This item was submitted to Loughborough's Institutional Repository (https://dspace.lboro.ac.uk/) by the author and is made available under the following Creative Commons Licence conditions.

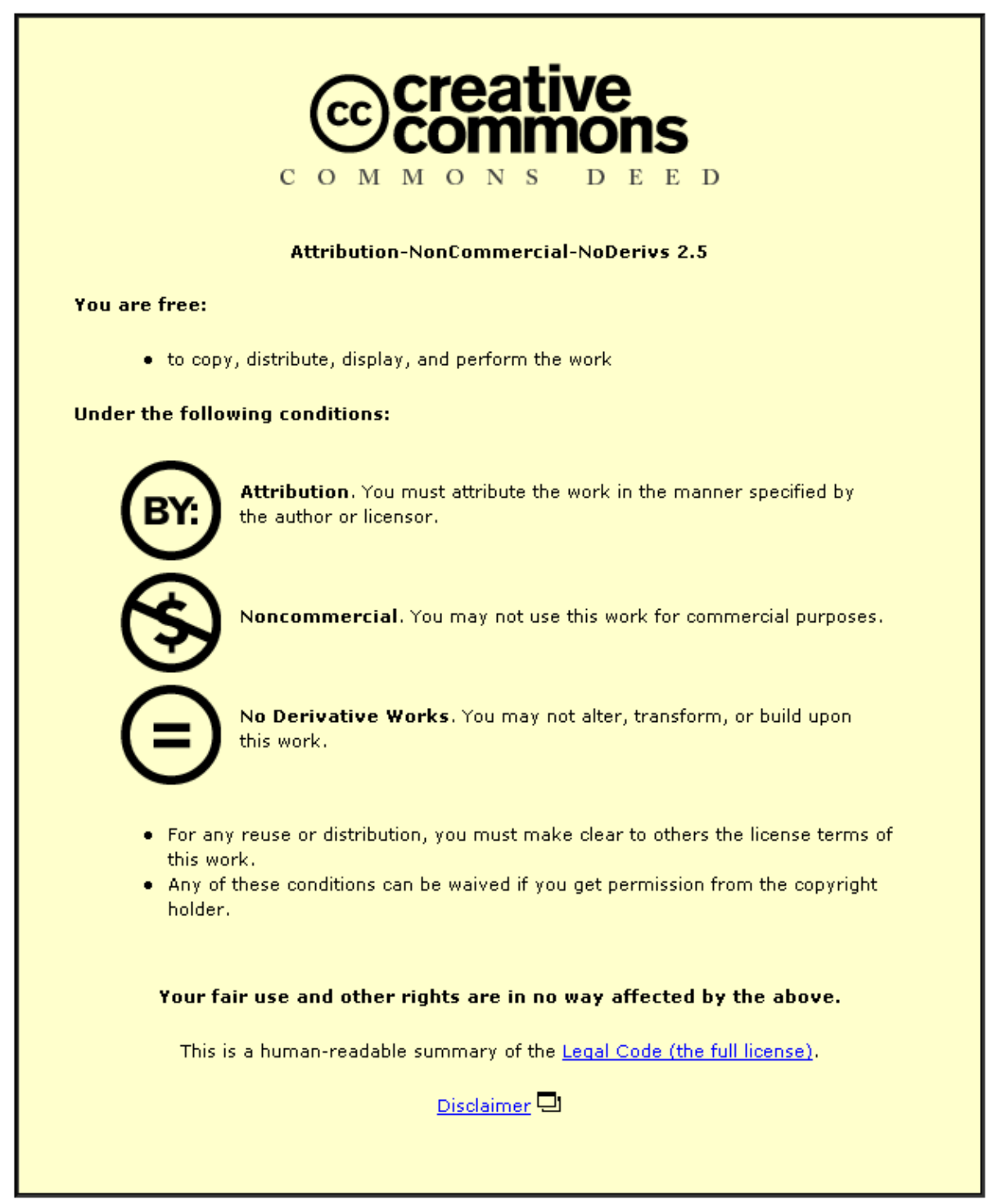

For the full text of this licence, please go to: http://creativecommons.org/licenses/by-nc-nd/2.5/ 


\title{
Swinging around the high bar
}

\author{
M.J. Hiley and M.R. Yeadon \\ Biomechanics Laboratory, \\ Department of Physical Education, Sports Science and Recreation Management, \\ Loughborough University, UK
}

\begin{abstract}
The motion of a gymnast around the high bar is modelled first as swinging around a rigid rod and then more accurately when the rod is considered to be elastic. The timing of the hip flexion and extension is explained as a consequence of the maximum torque limits.
\end{abstract}

\section{Introduction}

The high bar is one of six pieces of equipment used in Men's Artistic Gymnastics. The basic movement in competitive routines is the giant circle in which the gymnast tries to remain extended in the handstand position whilst circling the bar (Figure 1). The mechanics of this movement can be explained using simple models of the gymnast and the bar.

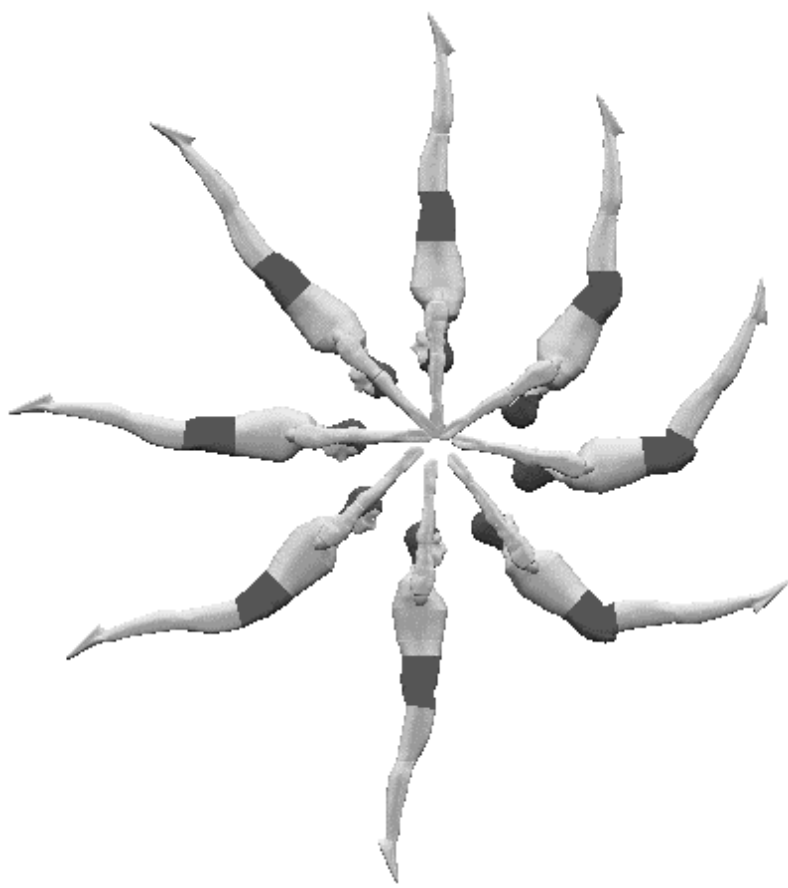

Figure 1. The backward giant circle on high bar. The gymnast circles from the handstand position above the bar in the anticlockwise direction.

\section{Conservation of energy}

Energy loses while circling the bar occur due to the friction between the hands and the bar but are relatively small. To a first approximation we can model the gymnast as a rigid rod swinging around a frictionless inelastic bar. The rigid rod is given a mass $m$, has its mass centre a distance $r$ from the bar and has moments of inertia about the bar and its mass centre ( $\mathrm{I}$ and $\mathrm{I}_{\mathrm{G}}$, respectively) similar to those of a gymnast in a handstand position. If the model is given an initial angular velocity $\omega_{0}$ the angular velocity $\omega_{1}$ as the gymnast passes beneath the bar may be calculated. 
Taking the acceleration due to gravity as $\mathrm{g}$ :

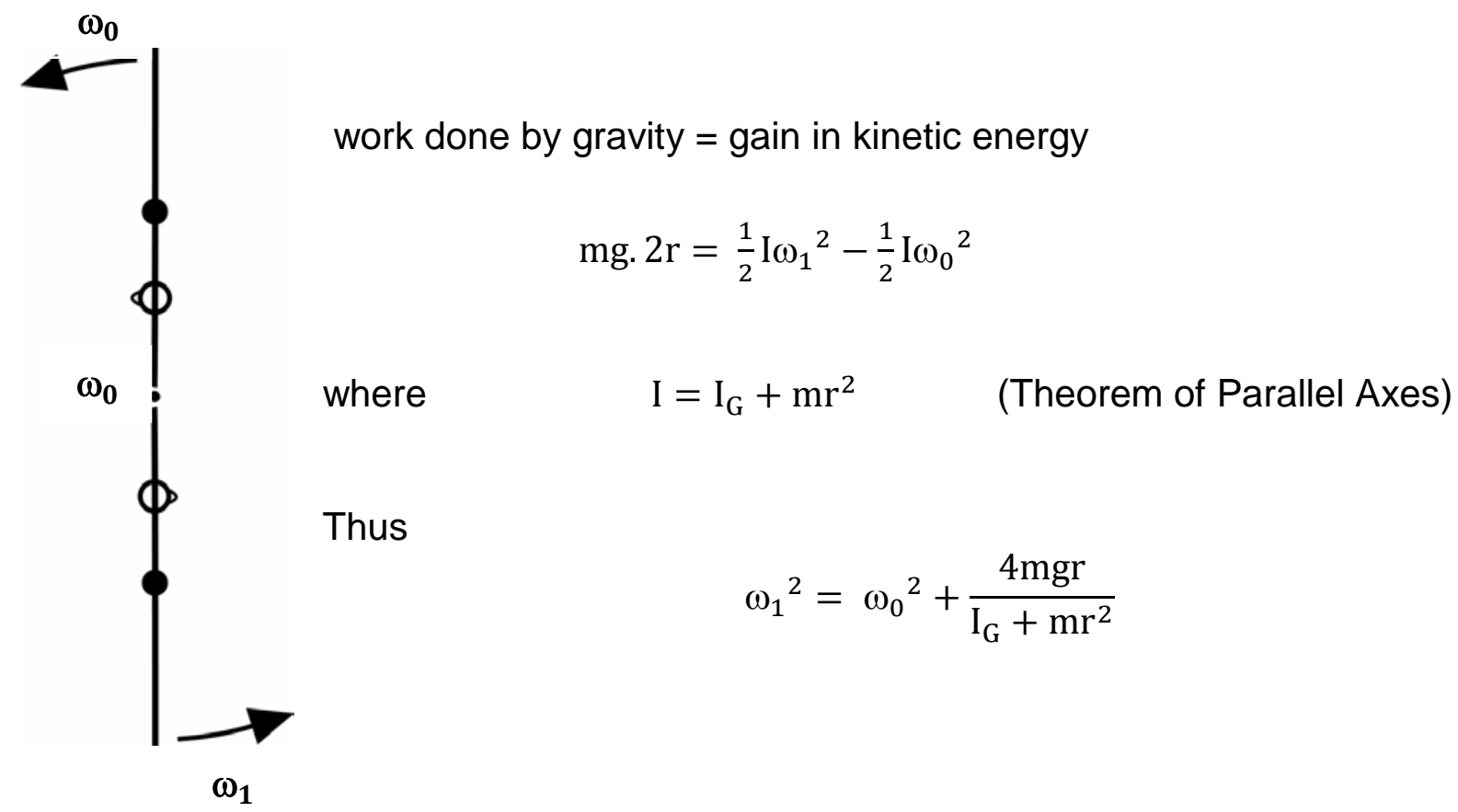

Figure 2. Single segment rigid model.

In the case of starting from a still handstand $\omega_{0}=0 \mathrm{rad} . \mathrm{s}^{-1}$ and taking $\mathrm{m}=60 \mathrm{~kg}$, $\mathrm{g}=9.81 \mathrm{~m} \cdot \mathrm{s}^{-1}, \mathrm{r}=1.0 \mathrm{~m}$ and $\mathrm{I}_{\mathrm{G}}=10 \mathrm{~kg} \cdot \mathrm{m}^{2}$ gives $\omega_{1}=5.8 \mathrm{rad} . \mathrm{s}^{-1}$ which is the correct order of magnitude although a little larger than measured values.

The reaction force $\mathrm{R}$ exerted on the hands can be calculated at the lowest point of the circle using Newton's Second Law:

$$
\mathrm{R}-\mathrm{mg}=\mathrm{mr} \omega_{1}{ }^{2}
$$

Here the term $r \omega_{1}{ }^{2}$ is the centripetal acceleration towards the centre of the circle described by the mass centre. Thus:

$$
\mathrm{R}=\mathrm{mg}+\operatorname{mr} \omega_{1}{ }^{2}=\mathrm{mg}\left[1+\frac{\mathrm{r} \omega_{1}{ }^{2}}{\mathrm{~g}}\right]=\operatorname{mg}[1+3.4]>4 \mathrm{mg}
$$

In other words the reaction force at the bar is in excess of $4 \mathrm{mg}$ which is somewhat larger than measured values.

\section{Elastic bar}

In the previous analysis it was assumed that the bar was inelastic. In practice the $28 \mathrm{~mm}$ diameter steel bars used by gymnasts deform by around $0.1 \mathrm{~m}$ at the lowest point of a giant circle.

Suppose that the bar deforms by a distance $h$ during the downswing. If the initial angular velocity is zero at the highest point the reaction force on the bar is $\mathrm{R}=\mathrm{mg}$ acting downwards. Suppose that this depresses the bar downwards through a distance $d_{1}$ from the neutral bar position. At the lowest point the downwards force 
on the bar will be:

$$
\mathrm{R}=\mathrm{mg}+\mathrm{m}\left(\mathrm{r}+\mathrm{d}_{2}\right) \omega_{2}{ }^{2}
$$

where $d_{2}$ is the depression from the neutral bar position.

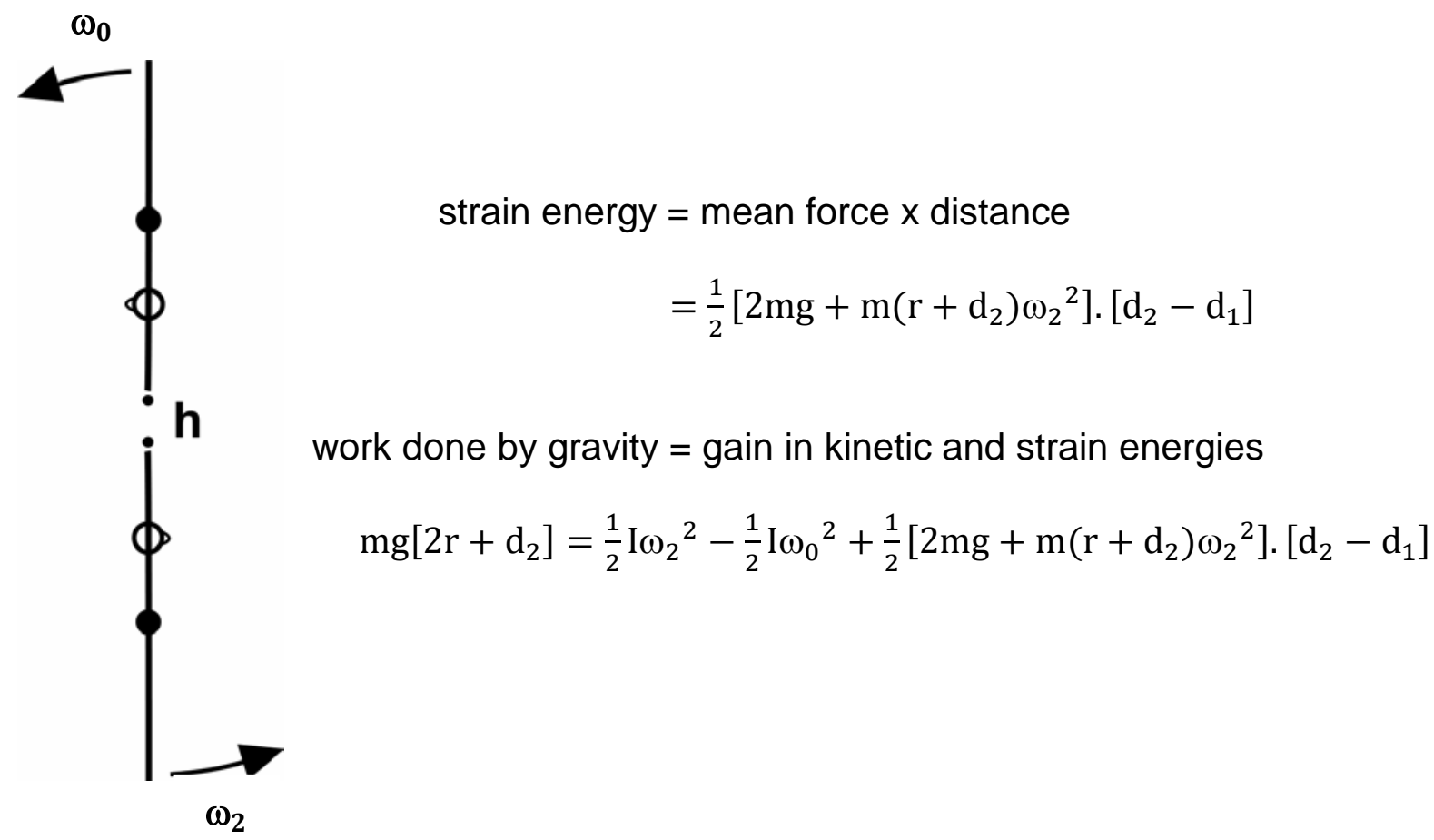

Figure 3. Single segment elastic model.

Suppose we estimate $d_{1}=0.02 \mathrm{~m}$ and $d_{2}=0.10 \mathrm{~m}$. At the lowest point this gives $\omega_{2}=5.2$ rad. $\mathrm{s}^{-1}$ and $\mathrm{R}=4.0 \mathrm{mg}$. These values are closer to the actual values of $\omega=5.0$ rads $^{-1}$ and $\mathrm{R}=3.3 \mathrm{mg}$ than the previous analysis with a rigid bar. Further improvement can be made by using an elastic model of the gymnast since gymnasts stretch by $0.15 \mathrm{~m}$ during a giant circle.

\section{Weightless}

Prior to a dismount the gymnast uses accelerated giant circles to build up angular velocity around the bar. This means that at the highest point the angular velocity can be quite large. If the angular velocity is large enough the gymnast will be "weightless" at the highest point. In other words the centripetal force is provided by the weight.

Newton's Second Law: $\mathrm{mg}-\mathrm{R}=\mathrm{mr}_{0}{ }^{2}$

When $\mathrm{R}=0, \mathrm{mg}=\mathrm{mr}_{0}{ }^{2}$ and $\omega_{0}=\sqrt{\mathrm{g}}=3.13 \mathrm{rad} . \mathrm{s}^{-1}$

This compares with a value of $2.5 \mathrm{rad} . \mathrm{s}^{-1}$ from an actual performance. 


\section{Accelerated circles}

In order to increase the angular velocity in successive giant circles it is necessary to put energy into the system. A simple point mass model was used by Bauer (1983) to explain how this can be done (Figure 4). The model assumes that the gymnast flexes instantaneously at the lowest point. By flexing the gymnast raises his mass centre and reduces the moment of inertia about the bar. The increase in energy due to flexing may be calculated from the increase in potential and kinetic energy. Using the same numbers as those in the first example, if the mass centre is raised $0.10 \mathrm{~m}$ at the lowest point and the moment of inertia about the bar is reduced to $60 \mathrm{~kg} \cdot \mathrm{m}^{2}$ from $70 \mathrm{~kg} \cdot \mathrm{m}^{2}$ :

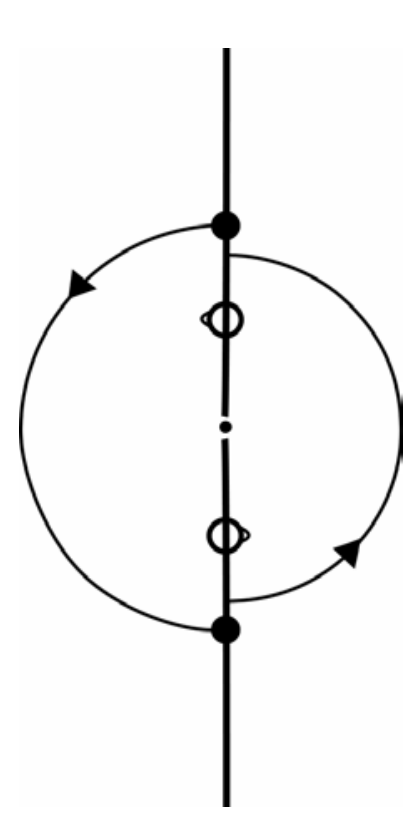

$$
\begin{aligned}
\text { increase in potential energy } & =\text { mgh } \\
& =60 \times 9.81 \times 0.10=58.9 \text { Joules }
\end{aligned}
$$

new angular velocity may be found using conservation of angular momentum

$$
\begin{aligned}
\mathrm{I}_{1} \omega_{1} & =\mathrm{I}_{2} \omega_{2} \\
\omega_{2} & =\frac{\mathrm{I}_{1} \omega_{1}}{\mathrm{I}_{2}}=6.8 \mathrm{rads}^{-1}
\end{aligned}
$$

increase in kinetic energy $=\frac{1}{2} \mathrm{I} \omega_{2}^{2}-\frac{1}{2} \mathrm{I} \omega_{1}^{2}=209.9$ Joules

$$
\begin{aligned}
\text { total increase in energy } & =\text { increase in potential energy } \\
& + \text { increase in kinetic energy } \\
& =268.7 \mathrm{~J}
\end{aligned}
$$

Figure 4. Bauer's (1983) point mass model of a gymnast performing backward giant circles.

Once the gymnast reaches the highest point an extension is performed. Again the potential energy is increased by raising the mass centre, although, the kinetic energy is reduced slightly due to the increase in moment of inertia about the bar. If the net increase in potential and kinetic energy during these actions is greater than the loss of energy due to friction, the gymnast will complete the giant circle with more kinetic energy and hence more angular velocity than he started with.

The same point mass model may used to describe the basic technique of the backward giant circle from the perspective of the torque created by the gymnast's weight. During the downswing the gymnast remains extended so as to maximise the effect of the torque, which increases the gymnast's angular velocity about the bar. During the upswing the torque created by the gymnast's weight slows the gymnast's angular velocity. To reduce the effect of the torque during the upswing the gymnast closes his hip and shoulder angles reducing the moment arm of the weight and hence the torque itself (Figure 5). 


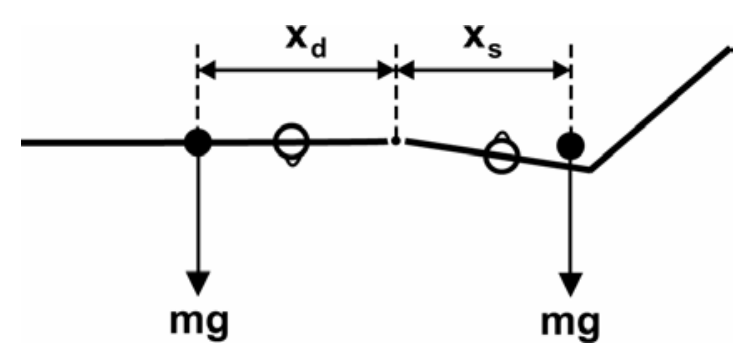

Figure 5. By moving the mass centre closer to the bar during the upswing the gymnast reduces the effect of the torque tending to reduce his angular velocity.

\section{Timing the flexion and extension}

According to Bauer's model the gymnast should flex and extend instantaneously at the lowest and highest points, respectively. In practice gymnasts flex after the lowest point and extend before they reach the highest point (Figure 1). Why is this the case?

A real gymnast comprises many segments, such as the arms, the torso and legs, which have their own moments of inertia. For the gymnast to change the angles between these segments he must exert joint torques. Suppose the gymnast was modelled using two rigid segments, the arms and torso forming one segment and the legs the other. As the model swings from the handstand position a joint torque tending to close the hip joint (hip flexor torque) is required to maintain an extended body shape. If there were no torque at the hip during the downswing the hip angle would open (hyper-extension at the hip). However, during the upswing a torque tending to open the hip angle (hip extensor torque) is now required to maintain an extended body shape. If during the upswing the torque at the hip were set to zero the model would flex at the hip.

Consider two hip flexions performed either side of the lowest point of the giant circle (Figure 6) with the same change in hip angle performed over the same duration. The flexion action performed before the lowest point will require a larger torque than the flexion performed after the lowest point so more concentric work is done and there will be a larger increase in energy. This is because at the lowest point the model would tend to close the hip joint if the torque was set to zero and so less work is required to produce the angle change. The reason gymnasts flex after the lowest point is due to the size of the joint torques that they are able to exert or choose to exert. There are two possibilities: either the gymnasts are not strong enough to flex before the lowest point or they choose to work within themselves.

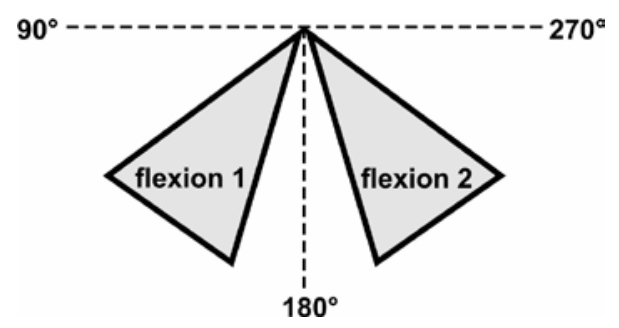

Figure 6. Two flexion actions performed either side of the lowest point of a giant circle. 
The same mechanics apply to the extension that the gymnast performs near the highest point. More concentric work is done by extending before the highest point, due to the larger torques that are required. If the gymnast were unable to exert this level of torque he would delay the extension until after the highest point. However, the joint torques involved with the extension are less than those involved with the flexion through the lowest point and so the gymnast is able to extend before the highest point producing a greater increase in energy.

More complex computer simulation models of swinging have been developed (Hiley, 1998) and are currently in use to analyse giant circles used by elite gymnasts at the Sydney Olympics.

\section{References}

Bauer, W.L. (1983). Swinging as a way of increasing the mechanical energy in gymnastic manoeuvres. In H. Matsui and K. Kobayashi (Eds.), Biomechanics VIII-B. Champaign, IL: Human Kinetics. pp. 801-806.

Hiley, M.J. (1998). Mechanics of the giant circle on high bar. Doctoral dissertation. Loughborough University. 